\title{
LA EQUIVALENCIA Y SUPRESIÓN EN LA RESOLUCIÓN DEL PROBLEMA INVERSO EN GEOELÉCTRICA: APLICACIÓN EN SANTA LUCÍA COTZUMALGUAPA, GUATEMALA
}

\author{
Ana Beatriz Cosenza Muralles ${ }^{1} \&$ Mario E. Arias Salguero ${ }^{2 *}$ \\ ${ }^{1}$ Centro de Estudios Superiores de Energía y Minas, \\ Edificio T1, $3^{\text {er }}$ nivel, Ciudad Universitaria, Z 12 \\ Universidad de San Carlos de Guatemala, Guatemala \\ ${ }^{2}$ Escuela Centroamericana de Geología, Apdo. 214-2060 \\ Universidad de Costa Rica, San José \\ *Autor para contacto: marioa@geologia.ucr.ac.cr
}

(Recibido: 27/10/06; aceptado: 07/03/07)

\begin{abstract}
In order to obtain a correct interpretation of a vertical electrical sounding (VES), the suppression and equivalence principles must be considered, for generating multiple models as a consequence of the non unicity of the solution of the inverse problem. Among all the valid solutions, one must be found that agrees the most with the VES nearby, that has a good geological meaning in function of the previous information of the site and that agrees with the hypothesis formulated during the research, just as it was done in the location of Santa Lucía Cotzumalguapa, Guatemala.

Keywords: Inverse problem, equivalence, suppression, vertical electrical sounding, Guatemala.

RESUMEN: Para una correcta interpretación de un sondeo eléctrico vertical (SEV), se deben considerar los principios de supresión y equivalencia, por los que se generan múltiples modelos como consecuencia de la no unicidad de la solución del problema inverso. Debe buscarse entre las soluciones válidas, aquella que armonice más con los SEV contiguos, que tenga un buen significado geológico en función de la información previa del sitio y que se enmarque dentro de la hipótesis planteada en la investigación, tal como se realizó en la localidad de Santa Lucía Cotzumalguapa, Guatemala.

Palabras clave: Problema inverso, equivalencia, supresión, sondeo eléctrico vertical, Guatemala.
\end{abstract}




\section{INTRODUCCIÓN}

El modelo numérico en el caso de la geofísica, es una representación matemática idealizada de una sección del subsuelo. Este modelo está constituido por un conjunto de parámetros, que son las cantidades físicas que se desea estimar a partir de los datos medidos. Por otra parte, la respuesta del modelo es el conjunto de datos sintéticos que puede calcularse a partir de las relaciones matemáticas que lo definen para el conjunto de parámetros dado. Se busca entonces un modelo numérico que produzca una respuesta similar a los datos medidos y que tenga una expresión geológica coherente.

En esencia, el método para obtener un modelo puede describirse de la siguiente manera (Menke, 1989):

Sea d un vector cuyas componentes d1, d2, ...dn son los datos medidos. En el caso del sondeo eléctrico vertical (SEV) estos datos son las resistividades aparentes y las distancias $\mathrm{AB} / 2$ (para el dispositivo Schlumberger).

Sea $\mathrm{m}$ un vector cuyas componentes $\mathrm{m} 1$, $\mathrm{m} 2$, ...mn son los parámetros desconocidos que se trata de calcular, en este caso, la resistividad real y el espesor de las capas en los SEV.

Si se conoce una solución aproximada mo, ya sea por un modelo de interpretación cualitativa a partir de asíntotas y tangentes, como el descrito en Telford et al. (1990) o con las curvas patrón, como el descrito por Orellana \& Mooney (1966), se puede estimar su grado de validez mediante el cálculo del vector $\mathrm{f}=\mathrm{f}(\mathrm{mo})$ que es la respuesta del modelo.

Además, se puede determinar la diferencia $\mathrm{g}=\mid \mathrm{d}-\mathrm{fl}$, entre el vector de datos medidos y los datos calculados, la cual puede ser minimizada por diversos métodos de optimización como el de mínimos cuadrados planteado por varios autores, entre ellos Tarantola \& Valette (1982 a, b), Loke \& Barker $(1995,1996)$ y el de gradiente máximo (Tarantola, 2005), cuyas soluciones deben ser estabilizadas por medio de métodos de regularización (Constable et al., 1987; Scales et al., 2001), como en el caso del método de mínimos cuadrados amortiguado.

La no unicidad del problema inverso es debida, según Tarnatola \& Valette (1982), por lo menos en algunos casos, al hecho de que los datos son discretos y no densos. Por ello, a cortes geológicos diferentes entre sí, pueden corresponder curvas de campo cuya discrepancia mutua es menor que el límite experimental de error, es decir: a cada curva de campo le corresponden $\mathrm{n}$ diferentes combinaciones de espesores y resistividades. Es por ello que en la teoría de medios conductores estratificados, los parámetros y funciones que resultan de productos y cocientes entre el espesor y la resistividad de cada capa del sondeo, cobran gran importancia.

Maillet (1947) propone los parámetros de Dar Zarrouk, que incluyen: conductancia longitudinal, resistencia transversal y la pseudo-anisotropía. Orellana (1972), Meyer de Stadelhofen (1995) y Reynolds (1997) entre otros, describen los fundamentos y procedimientos para su cálculo.

\section{La equivalencia y la supresión}

La solución al problema inverso de los sondeos eléctricos verticales (SEV) no es única; un mismo conjunto de datos de campo admite todo un dominio de soluciones válidas. El principio de equivalencia se refiere a las modificaciones que pueden efectuarse en una capa del corte geoeléctrico sin alterar la profundidad del techo de la capa, y existen dos posibles casos:

Cuando la resistencia transversal $\mathrm{T}=$ ge de una capa es mucho menor que la del conjunto de capas conductoras adyacentes, esta puede ser sustituida por otra de distinta resistividad (Q) y espesor (e) pero del mismo valor de $\mathrm{T}$, sin que el efecto del ajuste de la capa sobre el conjunto de interpretación haga que la curva de resistividad aparente varíe significativamente, tal como lo muestra la figura 1 , realizada a partir de la modelización directa y calculada a partir de los datos del cuadro 1 (Arias, 1999).

En el caso de que la conductancia longitudinal $\mathrm{S}=\mathrm{e} / \mathrm{Q}$ sea mucho menor que la del conjunto de capas resistivas adyacentes, la capa en cuestión puede ser sustituida por otra que mantenga una misma relación de $\mathrm{S}$. Tal como lo muestran el cuadro 2 y la figura 2, realizados a partir de la modelización directa (Arias, 1999). 


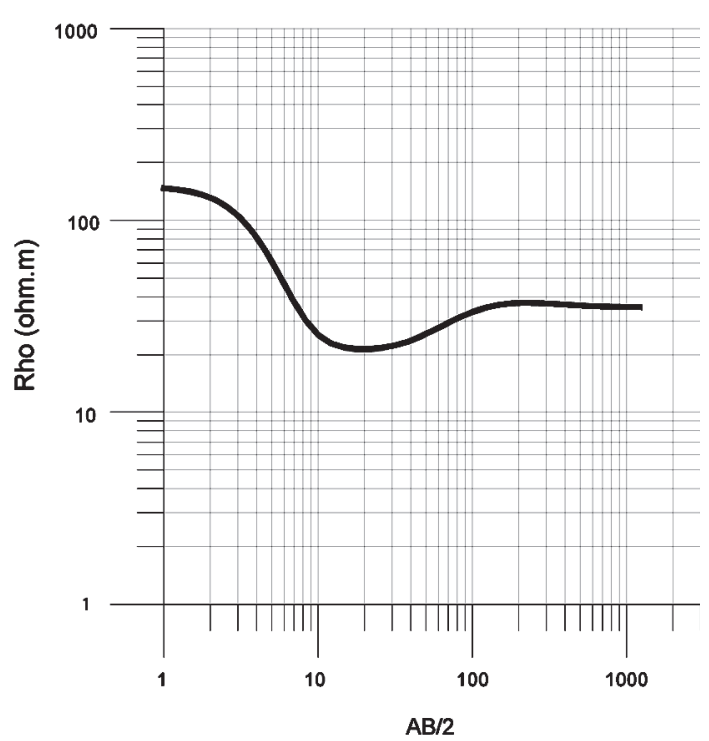

Fig. 1: Modelización directa del efecto de resistencia transversal.

Por su parte, el principio de supresión corresponde al cambio del número de capas sin afectar la curva de resistividad aparente. La figura 3, explica el caso de una capa cuya resistividad es intermedia entre las capas adyacentes, en el caso en que el espesor es muy grande, la influencia sobre la curva permite distinguirla con facilidad. Sin embargo, al ir disminuyendo el espesor, su influencia disminuye e incluso puede ser suprimida (capa ciega) y pasar inadvertida (cuadro 3). Parasnis (1997) considera y explica también el caso de una capa de espesor pequeño comparado

\section{Cuadro1}

Cálculo de la resistencia transversal de un modelo directo

\begin{tabular}{cccc}
\hline & $\begin{array}{c}\text { Resistividad } \\
\text { aparente } \\
(\Omega \mathrm{m})\end{array}$ & Espesor $(\mathrm{m})$ & $\mathrm{T}=\mathrm{\varrho e}$ \\
\hline \multirow{3}{*}{ Modelo1 } & 150 & 2 & - \\
& 20 & 33 & - \\
& 300 & 5 & 1500 \\
\cline { 2 - 4 } Modelo 2 & 35 & - & \\
& 150 & 2 & - \\
& 20 & 33 & - \\
& 125 & 12 & 1500 \\
\hline
\end{tabular}

Cuadro 2

Cálculo de la conductancia longitudinal de un modelo directo

\begin{tabular}{cccc}
\hline & $\begin{array}{c}\text { Resistividad } \\
\text { aparente } \\
(\Omega \mathrm{m})\end{array}$ & Espesor $(\mathrm{m})$ & $\mathrm{T}=\mathrm{Qe}$ \\
\hline Modelo 1 & 50 & 3 & - \\
& 150 & 25 & - \\
Modelo 2 & 5 & 6 & 1,2 \\
& 100 & - & - \\
\hline & 50 & 3 & - \\
& 150 & 25 & - \\
\hline
\end{tabular}

con la profundidad a la que se encuentra, donde su influencia en la curva de resistividad aparente resulta mínima.

Orellana (1972), discute otra fuente de ambigüedad, la seudo-anisotropía. En este caso, si en un corte estratigráfico existe una capa con anisotropía transversal (A) y espesor (e), esta se comporta, en lo que respecta a la distribución del potencial, como una capa isótropa de espesor e = Ae. Así, la presencia de capas anisótropas exagera

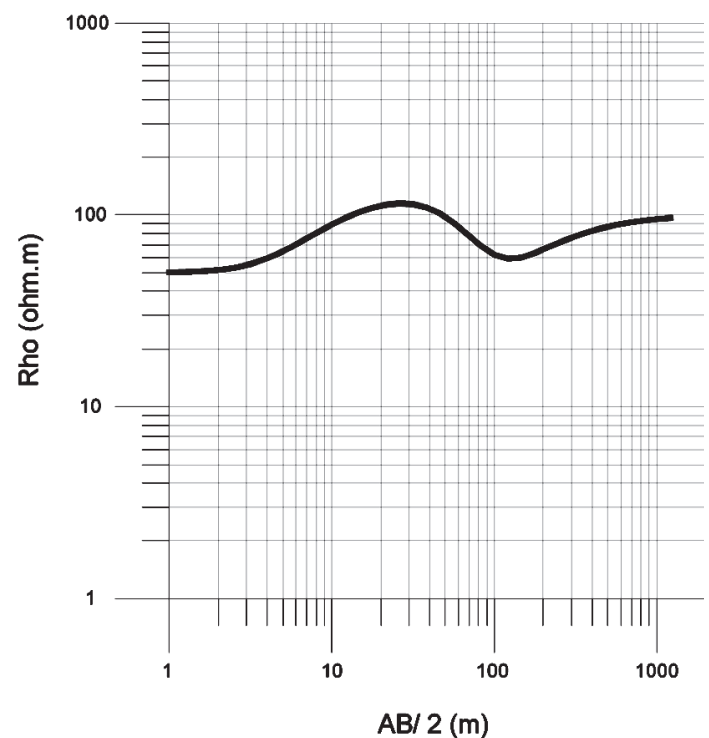

Fig. 2: Modelización directa del efecto de conductancia longitudinal. 


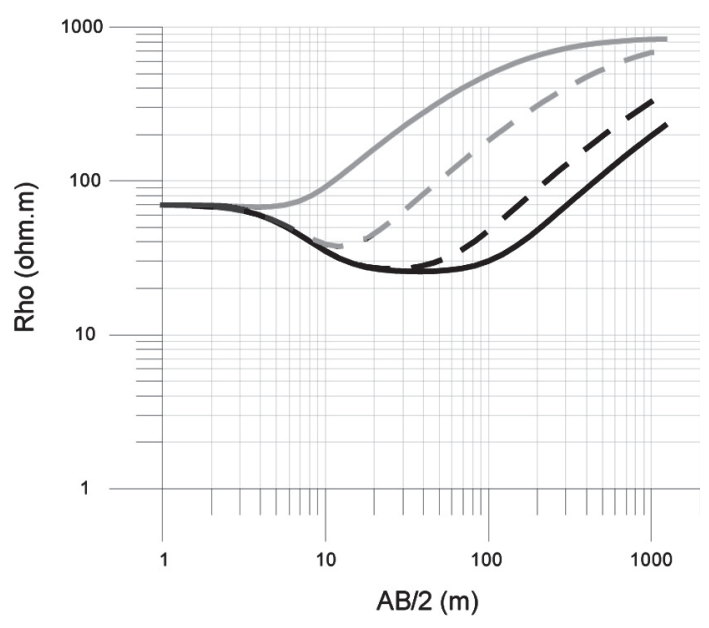

Fig. 3: Modelización directa del principio de supresión de una capa conductora ente dos resistivas. Línea continua negra e2 $=$ $100 \mathrm{~m}$; línea discontinua negra e $2=50 \mathrm{~m}$; línea discontinua gris e $2=10 \mathrm{~m}$ y línea continua gris e $2=1,5 \mathrm{~m}$.

la profundidad interpretada.

También hay que considerar los efectos superficiales, pues las heterogeneidades pueden producir distorsiones y ocultar estructuras profundas. Ritz et al. (1999) dividen estas distorsiones en dos grupos: los efectos causados por las heterogeneidades de superficie (HS) cercanas a un electrodo de corriente (efecto C) o a uno de potencial (efecto $\mathrm{P}$ ). Los efectos $\mathrm{C}$ en los sondeos eléctricos verticales distorsionan solo un segmento de la curva. Por otra parte, los efectos P desplazan la curva de resistividad aparente hacia arriba o hacia abajo sin cambiar su forma original.

Orellana (1972) consideró que los principios de equivalencia y de supresión generan cambios poco significativos en la forma de la curva del sondeo, sin embargo su influencia se ve reflejada en su interpretación.

\section{EJEMPLO DE APLICACIÓN}

Cosenza (2006) interpreta 5 SEV realizados en la finca El Baúl, localizada en el municipio de Santa Lucía Cotzumalguapa en el departamento de Escuintla, Guatemala (Fig. 4), en el marco de una investigación hidrogeofísica.

En la zona de estudio están reportadas dos unidades geológicas (IGN, 1993): por una parte, la "unidad de depósitos piroclásticos cuaternarios", constituida por depósitos sueltos o muy poco consolidados producto de la caída y del flujo de cenizas y que genera acuíferos multiestratos, debido a las variaciones de permeabilidad primaria (MAGA, 1991). Por otra parte, la "unidad de lavas y tobas terciarias", que comprende todo el complejo eruptivo eyectado durante el Neógeno, se caracteriza por su intensa fracturación y alto grado de permeabilidad, desarrollando generalmente acuíferos libres (MAGA, 1991).

En la finca El Baúl, existe un pozo perforado (Daho, 1975), que sirvió de parámetro para realizar la interpretación de los SEV. La figura 5 muestra la litología existente, observándose además, que el nivel de agua subterránea (N.E.) se encuentra a una profundidad de $10,5 \mathrm{~m}$.

Para la interpretación de los SEV se utilizó el programa de inversión QWSELN desarrollado por Tabbagh (2003). Los modelos geoeléctricos de los diferentes sondeos obtenidos con el programa se muestran en la figura 6 , existiendo una buena correlación entre ellos, pues el error varía entre $4,2 \%$ y $8,9 \%$.

Se obtuvieron los intervalos de confianza calculados a partir del conjunto de modelos cercanos al modelo establecido, cuyo error varíe dentro de un margen del 10\% sobre el error obtenido inicialmente. El resultado indica para una capa determinada,

Cuadro 3

El principio de supresión en función de la reducción del espesor de la segunda capa.

\begin{tabular}{cccccccccc}
\hline Modelo & & 1 & & 2 & 3 & & 4 \\
\hline & $\mathrm{a}(\mathrm{m})$ & $\mathrm{e}(\mathrm{m})$ & $\mathrm{a}(\mathrm{m})$ & $\mathrm{e}(\mathrm{m})$ & $\mathrm{a}(\mathrm{m})$ & $\mathrm{e}(\mathrm{m})$ & $\mathrm{a}^{(\mathrm{m})}$ & $\mathrm{e}(\mathrm{m})$ \\
\hline Capa 1 & 70 & 3 & 70 & 3 & 70 & 3 & 70 & 3 \\
Capa 2 & 25 & 100 & 25 & 50 & 25 & 10 & 25 & 1,5 \\
Capa 3 & 850 & - & 850 & - & 850 & - & 850 & - \\
\hline
\end{tabular}



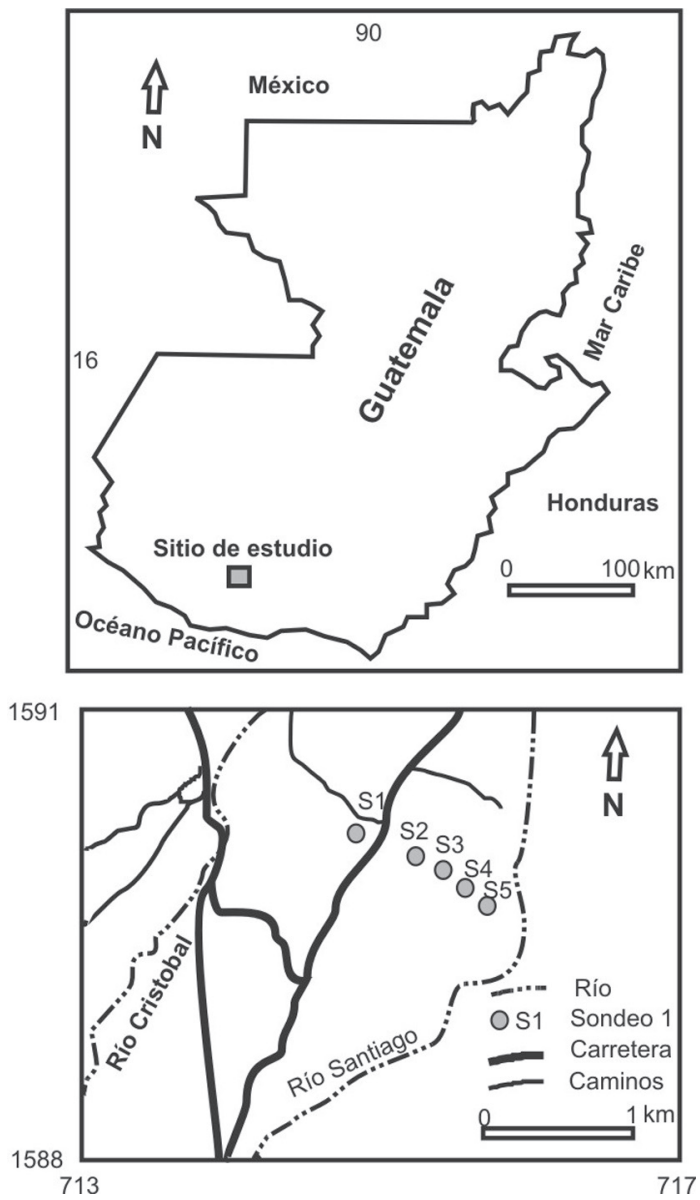

Fig. 4: Localización de los SEV en Santa Lucía de Cotzumalguapa.

el intervalo de valores permitido sin que se varíen los otros parámetros del modelo (Tabbagh, 2003). Los intervalos de variación para cada sondeo se muestran en los cuadros 4 y 5; las gráficas correspondientes en la figura 6 .

Las equivalencias de cada capa de los diversos sondeos se han calculado en los cuadros 6 y 7. Nótese que no se calculan para la última capa, pues es indeterminada en el espesor, ni para aquellas en que la cantidad de datos resulte insuficiente. Cuando los valores modelados están entre esos valores, se produce la equivalencia perfecta, es decir, se han determinado los valores de resistividad y espesor que producen la misma relación de conductancia longitudinal (S) y/o resistancia transversal ( $\mathrm{T}$ ).

A partir de los resultados de la inversión de cada SEV, se propone un modelo geoeléctrico, el cual correlaciona los valores de resistividad con la litología existente en la finca El Baúl. La figura 7 muestra el perfil de correlación en el cual la capa superficial con espesor que varía entre 0,4 y $1 \mathrm{~m}$, con valores de resistividad entre 150 y 450 $\Omega \mathrm{m}$, se asocia con suelo areno arcilloso; la segunda capa se caracteriza por tener un espesor entre 0,5 y $6,5 \mathrm{~m}$, resistividades entre los 1000 y $2000 \Omega \mathrm{m}$ y está asociada con arenas volcaniclásticas no saturadas. La tercera capa presenta espesores entre 2,5 y $25 \mathrm{~m}$, con resistividades entre los 100 y $230 \Omega \mathrm{m}$, asociadas con las arenas volcaniclásticas saturadas. Como basamento existe un sustrato relativamente conductor asociado con tobas arcillosas que presentan valores de resistividad entre 40 y $70 \Omega \mathrm{m}$. El SEV 2 logró

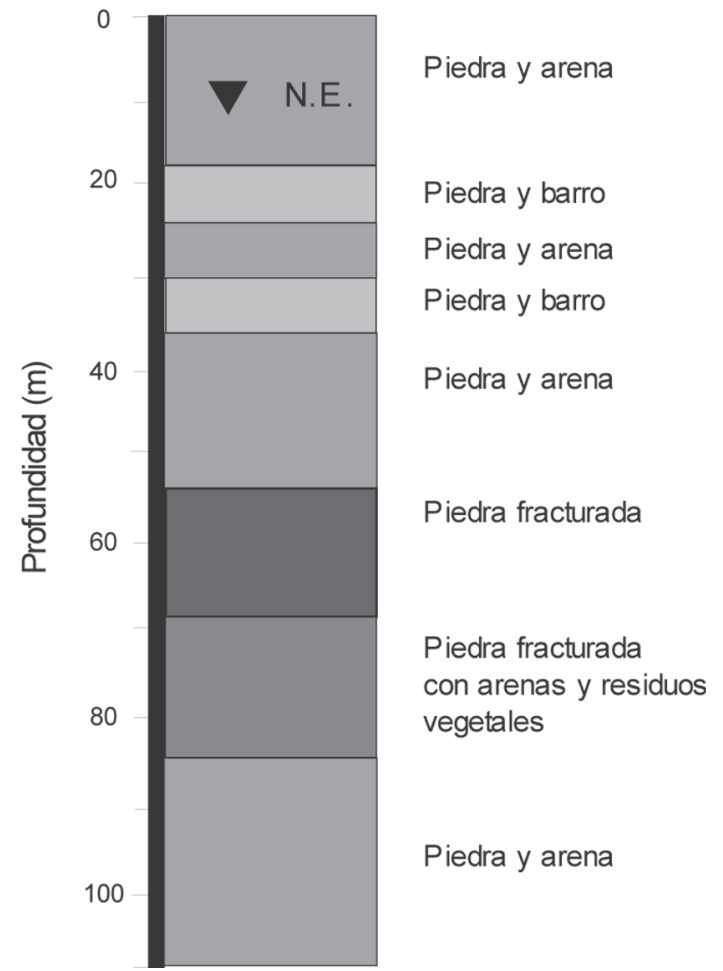

Fig.5: Litología del pozo localizado en fina El Baúl. 
Cuadro 4

Intervalos de confianza de la resistividad y espesor de los SEV1 y SEV2.

\begin{tabular}{|c|c|c|c|c|}
\hline Sondeo & \multicolumn{2}{|c|}{ SEV1 } & \multicolumn{2}{|c|}{ SEV2 } \\
\hline Error \% & \multicolumn{2}{|c|}{4,2} & \multicolumn{2}{|c|}{8,9} \\
\hline Capa & $\varrho(\Omega \mathrm{m})$ & $\mathrm{e}(\mathrm{m})$ & $\mathrm{\varrho}(\Omega \mathrm{m})$ & $\mathrm{e}(\mathrm{m})$ \\
\hline 1 & $\begin{array}{c}272,72- \\
330,00\end{array}$ & $0,72-0,88$ & $\begin{array}{c}118,18- \\
173,03\end{array}$ & $0,37-0,60$ \\
\hline 2 & $\begin{array}{c}1181,81- \\
1430,00\end{array}$ & $1.09-1,32$ & $\begin{array}{c}1181,81- \\
1903,60\end{array}$ & $0,41-0,73$ \\
\hline 3 & $\begin{array}{l}190,90- \\
231,00\end{array}$ & $\begin{array}{c}10,00- \\
12,10\end{array}$ & $\begin{array}{c}145,45- \\
193,60\end{array}$ & $\begin{array}{c}11,57- \\
15,40\end{array}$ \\
\hline 4 & $\begin{array}{c}63,63- \\
77,00\end{array}$ & - & $\begin{array}{c}33,05- \\
44,00\end{array}$ & $\begin{array}{c}45.45- \\
80,52\end{array}$ \\
\hline 5 & - & - & $\begin{array}{l}93,13- \\
181,50\end{array}$ & - \\
\hline
\end{tabular}

penetrar a una profundidad mayor a los $60 \mathrm{~m}$, asociándose el valor encontrado de resistividad $(150 \Omega \mathrm{m})$ con lavas.

\section{DISCUSIÓN Y CONCLUSIONES}

La finalidad del SEV es la determinación de la estructura del subsuelo a partir de dos etapas: la primera de ellas, consiste en obtener la distribución de la resistividad real del subsuelo; en la segunda, se busca el significado geológico de tales resistividades. La primera se basa en leyes físicomatemáticas, mientras que la segunda depende fundamentalmente de las correlaciones entre los datos y las observaciones geológicas.

La interpretación cuantitativa de un SEV es la resolución al problema inverso. Sin embargo, dado que la solución del problema inverso no es única, no basta con hallar una distribución vertical de las resistividades que produzca el menor porcentaje de error entre la curva de campo y el modelo propuesto. Más bien, se debe buscar entre las soluciones válidas, aquella que armonice más con los SEV contiguos, que tenga un buen significado geológico en función de la información previa del sitio y que se enmarque dentro de la hipótesis planteada en la investigación.

Es necesario contar en todos los casos con información geológica previa y realizar SEV paramétricos y multidireccionales.

Los SEV paramétricos son aquellos efectuados en las cercanías de un pozo o corte estratigráfico, donde se puedan conocer los espesores de las diferentes litologías existentes y modelar los valores de resistividad, con el fin de utilizarlos como parámetro para la interpretación de los sondeos adyacentes (considerando que hay homogeneidad en el valor de resistividad y posibles variaciones en el espesor de las capas).

Los SEV multidireccionales son varios SEV realizados con el mismo punto central, pero con diferente orientación en la abertura de los electrodos de medición (se pueden realizar dos o más SEV perpendiculares entre ellos), con el fin de poder validar la presunción requerida en la realización de todo SEV, que establece que el suelo debe ser horizontal, homogéneo e isótropo.

Cuadro 5

Intervalos de confianza de la resistividad y espesor de los SEV3, SEV4 y SEV5.

\begin{tabular}{ccccccc}
\hline Sondeo & \multicolumn{2}{c}{ SEV3 } & \multicolumn{2}{c}{ SEV4 } & \multicolumn{2}{c}{ SEV5 } \\
\hline Error $\%$ & \multicolumn{2}{c}{6,6} & & 5,4 & & 7,0 \\
\hline Capa & $\varrho(\Omega m)$ & $\mathrm{e}(\mathrm{m})$ & $\mathrm{Q}(\Omega \mathrm{m})$ & $\mathrm{e}(\mathrm{m})$ & $\mathrm{Q}(\Omega \mathrm{m})$ & $\mathrm{e}(\mathrm{m})$ \\
\hline 1 & $264,46-387,20$ & $0,33-0,44$ & $165,28-220,20$ & $0,72-0,96$ & $181,81-220,20$ & $0,68-0,82$ \\
2 & $909,09-1100,00$ & $0,54-0,66$ & $909,09-1100,00$ & $5,90-7,15$ & $1636,36-$ & $5,63-6,82$ \\
3 & $75,13-110,00$ & $1,12-2,90$ & $136,36-150,00$ & $1,57-18,63$ & $82,64-121,00$ & $19,53-38,06$ \\
4 & $54,54-66,00$ & - & $59,09-71,50$ & - & $54,54-72,60$ & - \\
\hline
\end{tabular}



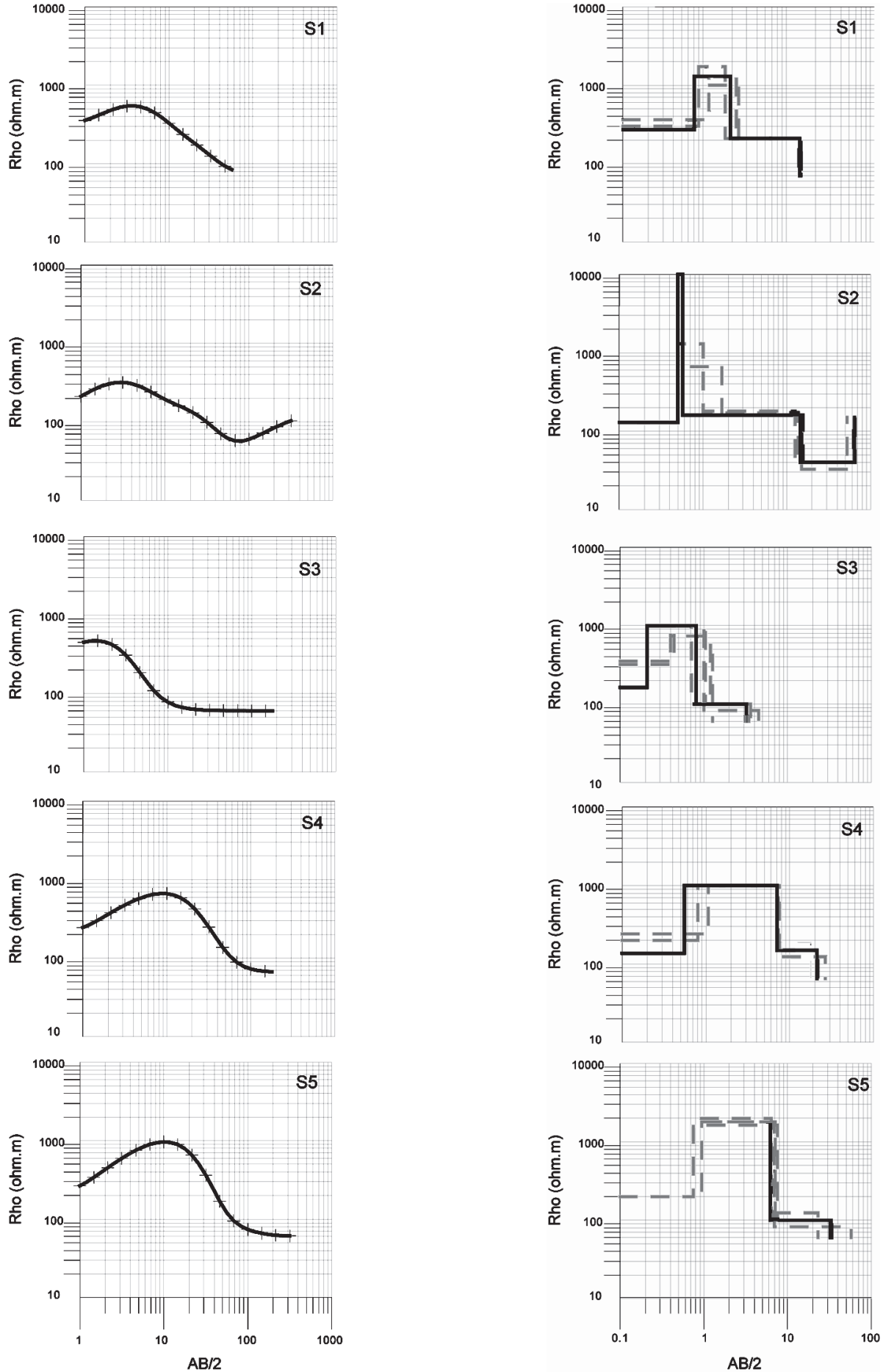

Fig. 6a: Modelos geoléctricos obtenidos con el QWSELN para cada SEV.
Fig. 6b: Intervalos de confianza obtenidos con el QWSELN para cada SEV. 


\section{Cuadro 6}

Equivalencias para los SEV1 y SEV2.

\begin{tabular}{ccccccc}
\hline Sondeo & \multicolumn{3}{c}{ SEV1 } & \multicolumn{5}{c}{ SEV2 } \\
\hline Capa & T & S & Correlación & T & S & Correlación \\
\hline 1 & - & 2,9 & 0,996 & - & - & - \\
2 & 1509,2 & - & -1 & 759,03 & - & $-0,973$ \\
3 & - & - & - & 466,04 & - & $-0,388$ \\
4 & - & - & - & - & 141,02 & 0,978 \\
\hline
\end{tabular}

Cuadro 7

Equivalencias para los SEV3, SEV4 y SEV5.

\begin{tabular}{cccccccccc}
\hline Sondeo & & SEV3 & \multicolumn{3}{c}{ SEV4 } & \multicolumn{3}{c}{ SEV5 } \\
\hline capa & $\mathrm{T}$ & $\mathrm{S}$ & $\mathrm{C}$ & $\mathrm{T}$ & $\mathrm{S}$ & $\mathrm{C}$ & $\mathrm{T}$ & $\mathrm{S}$ & $\mathrm{C}$ \\
\hline 1 & & 1,21 & 0,999 & & 4,35 & 0,999 & & 3,77 & 1 \\
2 & 576,07 & & -1 & & & & 13333 & $-0,998$ \\
3 & 157,08 & - & $-0,988$ & 157,08 & & $-0,988$ & 346,63 & $-0,987$ \\
\hline
\end{tabular}

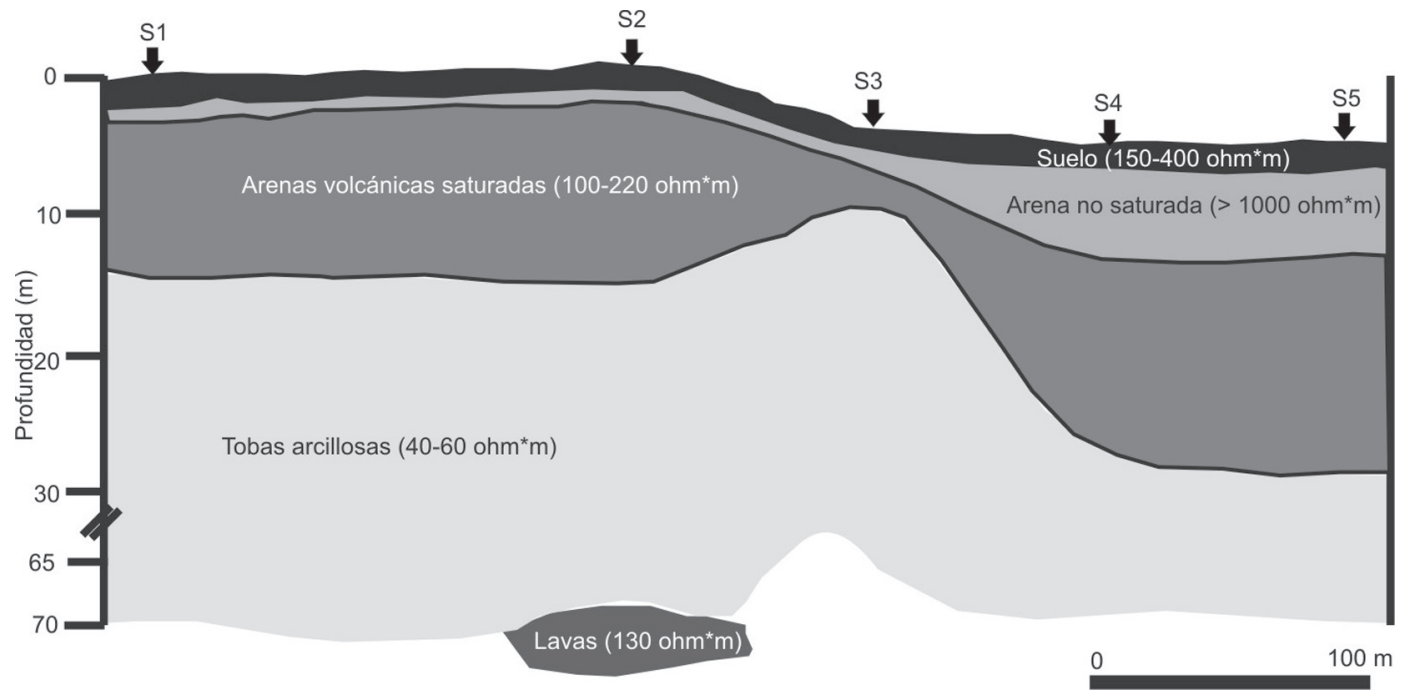

Fig. 7: Modelo geoeléctrico de la finca El Baúl.

Los modelos de inversión (resistividades y espesores) obtenidos para cada SEV de la finca El Baúl, validados a partir del análisis de confiabilidad y equivalencia de cada capa, así como de la correlación geológica permiten obtener un modelo geoeléctrico confiable, en el cual la zona productora de agua se encuentra a una profundidad que varía entre los 1 y $8 \mathrm{~m}$. 


\section{REFERENCIAS}

ARIAS, M.E., 1999: Les méthodes de sondages électriques et électromagnétiques: Examples d'application en Hydrogéologie.92 págs. Université Pierre et Marie Curie, Paris VI (Tesis de DEES).

CONSTABLE, S., PARKER, R. \& CONSTABLE, C., 1987: Occam's inversion: a practical algorithm for generating smooth models for electromagnetic sounding data .- Geophysics 53(3): 289-300.

COSEnZA, A. B., 2006: El sondeo eléctrico vertical como metodología geofísica en el estudio de capas acuíferas en la finca "El Baúl", Santa Lucía Cotzumalguapa.100 págs. Univ. de San Carlos de Guatemala (Tesis de Lic.).

DAHO POZOS DE CENTROAMÉRICA, S.A., 1975: Informe de perforación finca "El Baúl”. .- s.p Guatemala (Informe interno).

IGN, 1993: Mapa geológico de Guatemala, Hoja ND15-8-6 .- Escala: 1:250 000. IGN, Guatemala.

LOKE, M., \& BARKER, R., 1995: Least-squares deconvolution of apparent resistivity pseudosections .- Geophysics 60(6): 1682-1690.

LOKE, M., \& BARKER, R., 1996: Rapid leastsquares inversion of apparent resistivity pseudosections by a quasi Newton method.Geophysical Prospecting, 44: 131-142.

MAILLET, R., 1947: The fundamental equations of electrical prospecting .- Geophysics 12: 529-556.

MAGA, 1991: Plan maestro de riego y drenaje: Disponibilidad de agua subterránea.- 74 págs. Ministerio de Agricultura y Ganadería, Guatemala (Informe interno).
MENKE, W., 1989: Geophysical data analysis: discrete inverse theory.- 289 págs. Academic Press, California.

MEYER DE STADELHOFEN, C., 1995: Applications de la géophysique aux recherches d'eau .- 177 págs. Lavoisier, Paris.

ORELLANA, E.,1972: Prospección geoeléctrica en corriente continua .- 523 págs. Paraninfo, Madrid.

ORELLANA, E., \& MOONEY, H., 1966: Master tables and curves for Electrical Soundings.34 págs. Interciencia, Madrid.

PARASNIS, D., 1997: Principles of applied geophysics.- 429 págs. $5^{\text {a }}$ edición. Chapman \& Hall, Londres.

REYNOLDS, J., 1997: An Introduction to Applied and Environmental Geophysics .- 796 págs. Wyley, London.

RITZ, M., ROBAIN, H., PERVAGO, E., ALBOUY, Y., CAMERLYNCK, C., DESCLOITRES, M. \& MARIKO, A., 1999: Improvement to resistivity pseudosection modelling by removal of near-surface inhomogeneity effects: application to a soil system in south Cameroon.- Geophysical Prospecting 47: 85-101.

SCALES, J., SMITH, M. \& TREITEL, S., 2001: Introductory geophysical inverse theory.193 págs. Samizdat Press, Colorado.

TABBAGH, J., 2003: Documentation Qwseln, programme de sondage électrique.- 20 págs. Département de Géophysique Appliquée, Université Paris 6 (Informe interno).

TARANTOLA, A., 2005: Inverse problem theory and methods for model parameter estimation.- 342 págs. SIAM, Philadelphia. 
TARANTOLA, A., \& VALETTE, B., 1982a: Generalized non linear inverse problems solved using the least squares criterion.Reviews of Geophysics and Space Physics. 20(2): 219-232.
TARANTOLA, A., \& VALETTE, B., 1982b: Inverse problems $=$ quest for information.Journal of Geophysics, 50: 159-170.

TELFORD, W., GELDART, L. \& SHERIFF, R., 1990: Applied Geophysics.- 770 págs. Cambridge University Press, London. 